\title{
Preferable geometrical parameters of samples for piezooptic experiments
}

\author{
Kvasnyuk O., Vasylkiv Yu., Krupych O. and Vlokh R. \\ Vlokh Institute of Physical Optics, 23 Dragomanov Street, 79005 Lviv, Ukraine, \\ vlokh@ifo.lviv.ua
}

Received: 15.10 .2014

\begin{abstract}
We have shown that the errors of experimental determination of piezooptic coefficients, which are caused by friction appearing between sample surfaces and substrates can be eliminated by choosing properly geometrical parameters of the sample. The width-to-height ratio for the sample has to be equal or smaller than 1:3. In this case the error caused by a barrel-shaped distortion is reduced approximately to $1 \%$.
\end{abstract}

Keywords: piezooptic effect, inhomogeneous stresses, barrel-shaped distorsion

PACS: $78.20 . \mathrm{Hp}, 07.10 . \mathrm{Lw}$, 07.60.Fs

UDC: 535.012 .2

\section{Introduction}

Piezooptic (or photoelastic) effect is a well-known phenomenon of parametric optics [1-4]. The effect is successfully utilized in a lot of technical branches, e.g. in stress control and optical photoelastic tomography [4-10]. Perhaps the most important application of the piezooptic effect consists in operation of optical radiation via acoustooptic perturbation of refractive indices of an optical material $[11,12]$. As shown in our recent works (see e.g. [13]), the complete analysis of acoustooptic interaction and determination of the most efficient experimental geometries may be accomplished only when all of photoelastic tensor components are known. What is more, these components should be determined with high enough accuracies. The photoelastic coefficients $\left(p_{\lambda \mu}\right)$ can be directly determined in experiments using an acoustooptic Dixon-Cohen technique $[14,15]$. In spite of its high precision, this technique cannot reveal the sign of the photoelastic coefficients. The latter could be determined only after those coefficients are recalculated on the basis of relations $p_{\lambda \mu}=\pi_{\lambda \nu} C_{\nu \mu}$ (with $C_{\nu \mu}$ being the elastic stiffness tensor components and $\pi_{\lambda v}$ the piezooptic tensor components), provided that the piezooptic coefficients and the elastic modules are known in advance. Though the elastic modules are usually determined with a sufficient precision with the aid of a Papadakis technique [16], there remain notable problems with the accuracy of piezooptic coefficients.

Usually the piezooptic effect is studied with polarimetric or interferometric techniques. The both techniques are accurate enough with respect to the optical phase difference or the optical retardation. A problem appears only when one tries to determine mechanical stresses inside a loaded sample. As shown earlier in Ref. [17], the stress tensor components are non-uniformly distributed in the parallelepiped-shaped sample, even if a uniaxial compressing load is applied to a sample. The reason why a complicated spatial distribution of the stress components appears is misalignments of sample loading and a barrel-shaped distortion of a rectangular sample (see Fig. 1). So, the error in determination of the stress component $\sigma_{3}$ is about $30 \%$ if the sample is 
loaded, e.g., along its $X_{3}$ axis. This error can be reduced down to $\sim 14 \%$ by accurately aligning the sample in a pressure setup and carefully complying the condition of parallelism of the opposite sample surfaces. However, the barrel-shaped distortion, which appears due to friction forces arising between the upper and lower sample surfaces and the appropriate substrates, cannot be eliminated completely.

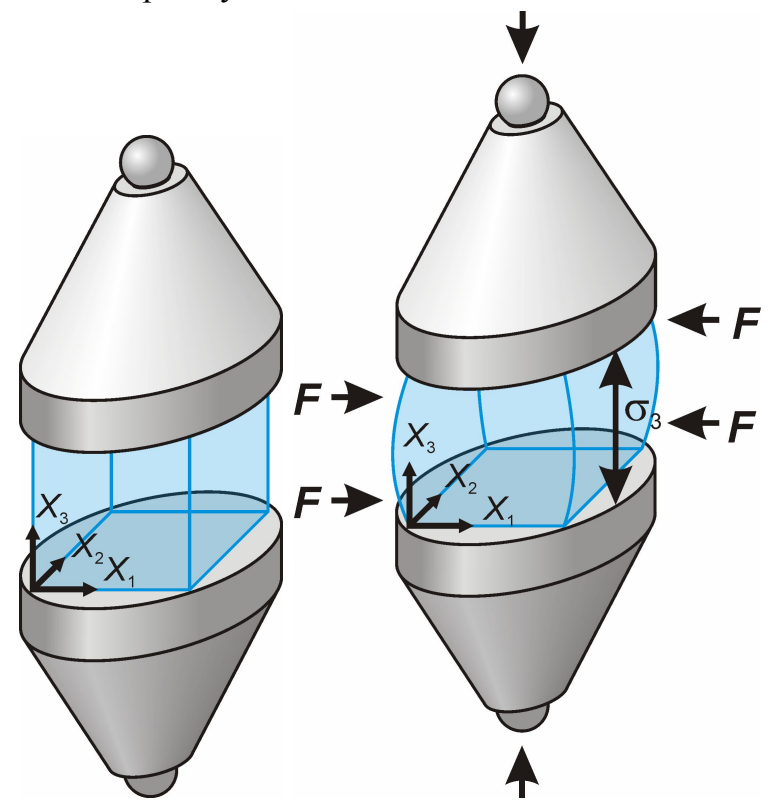

Fig. 1. Scheme of application of compressive loading, and a barrel-shaped distortion of sample appearing due to friction force $F$.

To increase the accuracy of piezooptic experiments, we have suggested a number of methods of sample loading producing spatially inhomogeneous distributions of the stress tensor components, of which coordinate dependences are known in advance. A four-point bending $[18,19]$ and a torsion of a crystalline bar $[20,21]$ are among these methods. They have turned out to be precise enough when determining the piezooptic coefficients. Moreover, some of them (e. g., a method of four-point bending) allow determining all of the piezooptic tensor components, at least in the easiest cases of isotropic or optically uniaxial crystals.

Nonetheless, the latter methods reveal a substantial practical disadvantage: they require a lot of samples with predetermined sizes and various crystallographic orientations. This necessitates growing of many (and large enough) crystalline boules. If one considers a possibility for turning again to simpler loading methods, the following questions appear: (i) Could the loading-related errors of the piezooptic experiments be minimized by optimizing a geometrical shape of a sample? and (ii) Which are those optimal geometrical parameters of the sample, first of all, the ratio of its width to its length, that make the errors caused by stress inhomogeneity inside a sample small, at least smaller than the errors typical for polarimetric or interferometric experiments themselves? The present work is devoted to solving of this problem on the basis of stress tensor distribution simulations in the isotropic glass samples.

\section{Simulations of stress distribution and optical phase difference in a sample}

Let us consider a parallelepiped-shaped isotropic BK7-glass sample with a square cross-section and different ratios of its width to height (1:1, 1:3 and 1:5). Let the compression load be applied along an $X_{3}$ axis (see Fig. 1). Assume that the loading force is uniformly distributed over the upper 
and lower surfaces and the substrates are covered by a layer of paper. Then the coefficient of friction between the glass and the paper is equal to $k=0.22$ (see Ref. [22]). Obviously, the friction forces appearing between the upper and lower sample faces and the substrates covered by paper should lead to a barrel-shaped distortion of the sample.

The maximum friction force $d F_{\max }$ is proportional to the loading stress, i.e. $d F_{\max }=k \sigma_{3} d S$, where $d S$ is the small element of square of the surface of the sample under load. The exact solutions of the equilibrium equations

$$
\frac{\partial \sigma_{v}}{\partial X_{j}}=0
$$

for the elastic body having a parallelepiped shape are as follows [23]:

$$
\begin{aligned}
& u=A_{0} \frac{\partial^{2} F_{1}^{0}}{\partial X_{1}^{2}}+\nabla^{2} F_{1}^{0}+A_{0} \frac{\partial^{2} F_{2}^{0}}{\partial X_{1} \partial X_{2}}-\frac{\partial f_{2}}{\partial X_{3}}+A_{0} \frac{\partial^{2} F_{3}^{0}}{\partial X_{1} \partial X_{3}}+\frac{\partial f_{3}}{\partial X_{1}}, \\
& v=A_{0} \frac{\partial^{2} F_{1}^{0}}{\partial X_{1} \partial X_{2}}+\frac{\partial f_{1}}{\partial X_{3}}+A_{0} \frac{\partial^{2} F_{2}^{0}}{\partial X_{2}^{2}}+\nabla^{2} F_{2}^{0}+A_{0} \frac{\partial^{2} F_{3}^{0}}{\partial X_{2} \partial X_{3}}-\frac{\partial f_{3}}{\partial X_{1}}, \\
& w=A_{0} \frac{\partial^{2} F_{1}^{0}}{\partial X_{1} \partial X_{3}}-\frac{\partial f_{1}}{\partial X_{2}}+A_{0} \frac{\partial^{2} F_{2}^{0}}{\partial X_{2} \partial X_{3}}+\frac{\partial f_{2}}{\partial X_{1}}+A_{0} \frac{\partial^{2} F_{3}^{0}}{\partial X_{3}^{2}}+\nabla^{2} F_{3}^{0} .
\end{aligned}
$$

Here $A_{0}=-\frac{\lambda+\mu}{\lambda+2 \mu}, \lambda, \mu$ are the Lame coefficients, $u, v, w$ the displacement vectors responsible for the mechanical strains, and $\nabla^{2}=\frac{\partial^{2}}{\partial x^{2}}+\frac{\partial^{2}}{\partial y^{2}}+\frac{\partial^{2}}{\partial z^{2}}$. Eqs. (2)-(4) involve the functions

$$
\begin{aligned}
& F_{1}^{0}=\sum_{n=1}^{\infty} \sum_{m=1}^{\infty}\left[K_{1}^{n m} \sinh \left(X_{1} r_{n m}\right)+K_{2}^{n m} \cosh \left(X_{1} r_{n m}\right)+K_{3}^{n m} X_{1} \sinh \left(X_{1} r_{n m}\right)\right. \\
& \left.+K_{4}^{n m} X_{1} \cosh \left(X_{1} r_{n m}\right)\right] \sin \frac{n \pi X_{2}}{b} \sin \frac{m \pi X_{3}}{c}, \quad r_{n m}=\pi \sqrt{\frac{n^{2}}{b^{2}}+\frac{m^{2}}{c^{2}}} \\
& F_{2}^{0}=\sum_{k=1}^{\infty} \sum_{m=1}^{\infty}\left[L_{1}^{k m} \sinh \left(X_{2} r_{k m}\right)+L_{2}^{k m} \cosh \left(X_{2} r_{k m}\right)+L_{3}^{k m} X_{2} \sinh \left(X_{2} r_{k m}\right)\right. \\
& \left.+L_{4}^{k m} X_{2} \cosh \left(X_{2} r_{k m}\right)\right] \sin \frac{k \pi X_{1}}{a} \sin \frac{m \pi X_{3}}{c}, \quad r_{k m}=\pi \sqrt{\frac{k^{2}}{a^{2}}+\frac{m^{2}}{c^{2}}}, \\
& F_{3}^{0}=\sum_{k=1}^{\infty} \sum_{n=1}^{\infty}\left[M_{1}^{k n} \sinh \left(X_{3} r_{k n}\right)+M_{2}^{k n} \cosh \left(X_{3} r_{k n}\right)+M_{3}^{k n} X_{3} \sinh \left(X_{3} r_{k n}\right)\right. \\
& \left.+M_{4}^{k n} X_{3} \cosh \left(X_{3} r_{k n}\right)\right] \sin \frac{k \pi X_{1}}{a} \sin \frac{n \pi X_{2}}{b}, \quad \sqrt{\frac{k^{2}}{a^{2}}+\frac{n^{2}}{b^{2}}}, \\
& f_{1}=\sum_{n=0}^{\infty} \sum_{m=0}^{\infty}\left[K_{5}^{n m} \sinh \left(X_{1} r_{n m}\right)+K_{6}^{n m} \cosh \left(X_{1} r_{n m}\right)\right] \cos \frac{n \pi X_{2}}{b} \cos \frac{m \pi X_{3}}{c}, \\
& f_{2}=\sum_{k=0}^{\infty} \sum_{m=0}^{\infty}\left[L_{5}^{k m} \sinh \left(X_{2} r_{k m}\right)+L_{6}^{k m} \cosh \left(X_{2} r_{k m}\right)\right] \cos \frac{k \pi X_{1}}{a} \cos \frac{m \pi X_{3}}{c},
\end{aligned}
$$




$$
f_{3}=\sum_{k=0}^{\infty} \sum_{n=0}^{\infty}\left[M_{5}^{k n} \sinh \left(X_{3} r_{k n}\right)+M_{6}^{k n} \cosh \left(X_{3} r_{k n}\right)\right] \cos \frac{k \pi X_{1}}{a} \cos \frac{n \pi X_{2}}{b},
$$

where $a, b$ and $c$ denote respectively the width, the length and the height of the parallelepiped sample ( $a=b$ in our case), and $K_{i}^{n m}, L_{i}^{k m}, M_{i}^{k n}$ are the coefficients that have to be determined.

It is evident that, by choosing in appropriate manner the above coefficients, one can select one or another boundary condition at the parallelepiped faces and describe different types of inhomogeneities. Notice that Bayda [23] has generalized this approach for the case of anisotropic parallelepipeds. Here the displacement vectors are as follows:

$$
\begin{aligned}
& u=\sum_{n=1}^{\infty} \sum_{m=1}^{\infty} \Theta_{1}^{n m}\left(X_{1}\right) \sin \frac{n \pi X_{2}}{b} \sin \frac{m \pi X_{3}}{c}+\sum_{k=0}^{\infty} \sum_{m=1}^{\infty} \Theta_{2}^{k m}\left(X_{2}\right) \cos \frac{k \pi X_{1}}{a} \sin \frac{m \pi X_{3}}{c}, \\
& +\sum_{k=0}^{\infty} \sum_{n=1}^{\infty} \Theta_{3}^{k n}\left(X_{3}\right) \cos \frac{k \pi X_{1}}{a} \sin \frac{n \pi X_{2}}{b} \\
& v=\sum_{n=0}^{\infty} \sum_{m=1}^{\infty} \Theta_{4}^{n m}\left(X_{1}\right) \cos \frac{n \pi X_{2}}{b} \sin \frac{m \pi X_{3}}{c}+\sum_{k=1}^{\infty} \sum_{m=1}^{\infty} \Theta_{5}^{k m}\left(X_{2}\right) \sin \frac{k \pi X_{1}}{a} \sin \frac{m \pi X_{3}}{c} \\
& +\sum_{k=1}^{\infty} \sum_{n=0}^{\infty} \Theta_{6}^{k n}\left(X_{3}\right) \sin \frac{k \pi X_{1}}{a} \cos \frac{n \pi X_{2}}{b}, \\
& w=\sum_{n=1}^{\infty} \sum_{m=0}^{\infty} \Theta_{7}^{n m}\left(X_{1}\right) \sin \frac{n \pi X_{2}}{b} \cos \frac{m \pi X_{3}}{c}+\sum_{k=1}^{\infty} \sum_{m=0}^{\infty} \Theta_{8}^{k m}(y) \sin \frac{k \pi X_{1}}{a} \cos \frac{m \pi X_{3}}{c} \\
& +\sum_{k=1}^{\infty} \sum_{n=1}^{\infty} \Theta_{9}^{k n}\left(X_{3}\right) \sin \frac{k \pi X_{1}}{a} \sin \frac{n \pi X_{2}}{b}
\end{aligned}
$$

The components of the mechanical stress tensor may be determined using the relations

$$
\begin{aligned}
& \sigma_{1}=2 \mu \frac{\partial u}{\partial X_{1}}+\lambda\left(\frac{\partial u}{\partial X_{1}}+\frac{\partial v}{\partial X_{2}}+\frac{\partial w}{\partial X_{3}}\right) \\
& \sigma_{2}=2 \mu \frac{\partial v}{\partial X_{2}}+\lambda\left(\frac{\partial u}{\partial X_{1}}+\frac{\partial v}{\partial X_{2}}+\frac{\partial w}{\partial X_{3}}\right), \\
& \sigma_{3}=2 \mu \frac{\partial w}{\partial X_{3}}+\lambda\left(\frac{\partial u}{\partial X_{1}}+\frac{\partial v}{\partial X_{2}}+\frac{\partial w}{\partial X_{3}}\right), \\
& \sigma_{4}=\mu\left(\frac{\partial w}{\partial X_{2}}+\frac{\partial v}{\partial X_{3}}\right) \\
& \sigma_{5}=\mu\left(\frac{\partial w}{\partial X_{1}}+\frac{\partial u}{\partial X_{3}}\right) \\
& \sigma_{6}=\mu\left(\frac{\partial u}{\partial X_{2}}+\frac{\partial v}{\partial X_{1}}\right) .
\end{aligned}
$$

Eqs. (5)-(10) may be simplified when the stress distribution becomes symmetric with respect to the mirror planes $X_{3}=c / 2, X_{1}=X_{2}$ and $X_{2}=-X_{1}+1$ : 


$$
\begin{aligned}
F_{1}^{0}= & \sum_{n=1}^{\infty} \sum_{m=1}^{\infty}\left\{K_{1}^{n m} \sinh \left[\left(X_{1}-\frac{a}{2}\right) r_{n m}\right]+K_{4}^{n m}\left(X_{1}-\frac{a}{2}\right) \cosh \left[\left(X_{1}-\frac{a}{2}\right) r_{n m}\right]\right\}, \\
& \times \sin \frac{n \pi X_{2}}{b} \sin \frac{m \pi X_{3}}{c} \\
F_{2}^{0}= & \sum_{k=1}^{\infty} \sum_{m=1}^{\infty}\left\{L_{1}^{k m} \sinh \left[\left(X_{2}-\frac{b}{2}\right) r_{k m}\right]+L_{4}^{k m}\left(X_{2}-\frac{b}{2}\right) \cosh \left[\left(X_{2}-\frac{b}{2}\right) r_{k m}\right]\right\}, \\
& \times \sin \frac{k \pi X_{1}}{a} \sin \frac{m \pi X_{3}}{c} \\
F_{3}^{0}= & \sum_{k=1}^{\infty} \sum_{n=1}^{\infty}\left\{M_{1}^{k n} \sinh \left[\left(X_{3}-\frac{c}{2}\right) r_{k n}\right]+M_{4}^{k n}\left(X_{3}-\frac{c}{2}\right) \cosh \left[\left(X_{3}-\frac{c}{2}\right) r_{k n}\right]\right\}, \\
& \times \sin \frac{k \pi X_{1}}{a} \sin \frac{n \pi X_{2}}{b} \\
& f_{1}=\sum_{n=0}^{\infty} \sum_{m=0}^{\infty} K_{6}^{n m} \cosh \left[\left(X_{1}-\frac{a}{2}\right) r_{n m}\right] \cos \frac{n \pi X_{2}}{b} \cos \frac{m \pi X_{3}}{c}, \\
& f_{2}=\sum_{k=0}^{\infty} \sum_{m=0}^{\infty} L_{6}^{k m} \cosh \left[\left(X_{2}-\frac{b}{2}\right) r_{k m}\right] \cos \frac{k \pi X_{1}}{a} \cos \frac{m \pi X_{3}}{c}, \\
& f_{3}=\sum_{k=0}^{\infty} \sum_{n=0}^{\infty} M_{6}^{k n} \cosh \left[\left(X_{3}-\frac{c}{2}\right) r_{k n}\right] \cos \frac{k \pi X_{1}}{a} \cos \frac{n \pi X_{2}}{b} .
\end{aligned}
$$

The symmetric distribution of stresses leads to the following relationships among the coefficients:

$$
K_{1,4}^{t m}=L_{1,4}^{t m}, M_{1,4}^{k n}=M_{1,4}^{n k}, K_{6}^{t m}=-L_{6}^{t m}, M_{6}^{k n}=-M_{6}^{n k} .
$$

Here we remind that the coordinate axes $X_{1}, X_{2}$ and $X_{3}$ are parallel to parallelepiped edges $a, b$, and $c$, respectively.

The exact boundary conditions are given by

$$
\left.\sigma_{1}\right|_{X_{1}=0}=0,\left.\sigma_{5}\right|_{X_{1}=0}=0,\left.\sigma_{6}\right|_{X_{1}=0}=0,\left.\sigma_{3}\right|_{X_{3}=0}=-1,\left|\sigma_{4}^{\max }\right|_{X_{3}=0}=k\left|\sigma_{5}^{\max }\right|_{X_{3}=0}=k \text {. }
$$

To simplify the procedure for obtaining the solutions of the stress components, we have accepted the condition $\sigma_{3}=-1$ at the lateral faces, since in the absence of friction forces the stress components should be given by

$$
\sigma_{3}=-1, \quad \sigma_{1}=0, \sigma_{2}=0, \sigma_{4}=0, \sigma_{5}=0, \sigma_{6}=0,
$$

which hold true for all of the sample volume. When the friction forces are present, the approximate boundary conditions read as

$$
\left.\sigma_{3}\right|_{X_{1}=0}=-1,\left.\sigma_{1}\right|_{X_{1}=0}=\left.0 \quad \sigma_{2}\right|_{X_{1}=0}=0,\left.\sigma_{3}\right|_{X_{3}=0}=-1,\left.\sigma_{1}\right|_{X_{3}=0}=-k,\left.\sigma_{2}\right|_{X_{3}=0}=-k .
$$

Below we will show that the approximate boundary conditions (29) yield in the solutions which agree well with the exact conditions (27). We have accepted that the number of harmonics is given by $n, m, k=1 . .59$. The relevant even terms can be neglected due to the symmetry of the stressed state.

Having obtained the distributions for all of the stress tensor components, we have simulated the phase difference for the case of light propagating along the $X_{2}$ axis. The sample has been divided into 10 layers $(l=1-10)$, which are mechanically and optically homogeneous along the 
light beam direction. Each of the layers has been divided by $i \times j=10 \times 10$ cells. The phase difference and the orientation of optical indicatrix have been simulated using the Jones matrix approach. The Jones matrix for each homogeneous cell may be written as

$$
J_{l}^{i j}=\left|\begin{array}{cc}
\left.e^{i \Delta \Gamma_{l}^{i j} / 2} \cos ^{2} \xi_{l}^{i j}+e^{-i \Delta \Gamma_{l}^{i j} / 2} \sin ^{2} \xi_{l}^{i j}\right) & i \sin \left(\Delta \Gamma_{l}^{i j} / 2\right) \sin \left(2 \xi_{l}^{i j}\right) \\
i \sin \left(\Delta \Gamma_{l}^{i j} / 2\right) \sin 2 \xi_{l}^{i j} & \left(e^{-i \Delta \Gamma_{l}^{i j} / 2} \cos ^{2} \xi_{l}^{i j}+e^{i \Delta \Gamma_{l}^{i j} / 2} \sin ^{2} \xi_{l}^{i j}\right)
\end{array}\right|,
$$

where the phase difference is determined by

$$
\Delta \Gamma_{l}^{i j}=\frac{2 \pi d_{l}}{\lambda}\left\{\frac{1}{2} n_{0}^{3}\left(\pi_{11}-\pi_{12}\right)\left(\left(\sigma_{1}\right)_{l}^{i j}-\left(\sigma_{3}\right)_{l}^{i j}\right) \sqrt{1+\left(\frac{2\left(\sigma_{5}\right)_{l}^{i j}}{\left(\sigma_{1}\right)_{l}^{i j}-\left(\sigma_{3}\right)_{l}^{i j}}\right)^{2}}\right\} .
$$

Here $d_{l}=10^{-3} \mathrm{~m}$ denotes the thickness of the homogeneous cells along the $X_{2}$ direction, and we have $n_{0}^{3}\left(\pi_{11}-\pi_{12}\right)=5.53 \times 10^{-12} \mathrm{~m}^{2} / \mathrm{N}$ for the light wavelength $\lambda=632.8 \mathrm{~nm}$ (see Krupych O. et al Ukr.J.Phys.Opt. 2011, 12, 150). The angle of optical indicatrix rotation around the $X_{2}$ axis is given by the relation

$$
\xi_{l}^{i j}=\frac{1}{2} \arctan \frac{2\left(\sigma_{5}\right)_{l}^{i j}}{\left(\sigma_{1}\right)_{l}^{i j}-\left(\sigma_{3}\right)_{l}^{i j}} .
$$

The resultant Jones matrix for the case when the beam propagates through all the layers is as follows:

$$
J^{i j}=\prod_{l=1}^{10} J_{l}^{i j}
$$

This matrix is involved in the relation

$$
\left[\begin{array}{l}
E_{1}^{\text {out }} \\
E_{3}^{\text {out }}
\end{array}\right]=J^{i j}\left[\begin{array}{l}
E_{1}^{\text {in }} \\
E_{3}^{\text {in }}
\end{array}\right]
$$

which links the amplitude components of the incident ( $E_{1}^{\text {in }}$ and $E_{3}^{\text {in }}$ ) and the outgoing ( $E_{1}^{\text {out }}$ and $\left.E_{3}^{\text {out }}\right)$ optical waves. Finally, the incident wave has been taken to be circularly polarized:

$$
\left[\begin{array}{l}
E_{1}^{i n} \\
E_{3}^{i n}
\end{array}\right]=\frac{1}{\sqrt{2}}\left[\begin{array}{l}
i \\
1
\end{array}\right] \text {. }
$$

\section{Results and discussion}

Fig. 2 to Fig. 4 display the main results of our simulations performed for the stress tensor components appearing in the parallelepiped-shaped isotropic glass samples with the square crosssections and different width-to-height ratios (1:1, 1:3 and 1:5). For the sample of cubic shape (i.e., the ratio 1:1), the stress tensor components $\sigma_{1}$ and $\sigma_{2}$ change from -0.013 in the central plane $X_{1} X_{2}$ (at $X_{3}=0.5 c$ ) to -0.085 at $X_{3}=0.2 c$ and $0.8 c$ (see Fig. 2a, b). The component $\sigma_{3}$ reaches its maximum equal to -1.12 in the sample centre and decreases in its absolute value down to -1.088 at $X_{3}=0.2 c$ and $0.8 c$ (see Fig. 2c). This means that the module of the actual $\sigma_{3}$ component observed in the sample centre is $12 \%$ higher than the supposed stress which has been created in the experiment. 

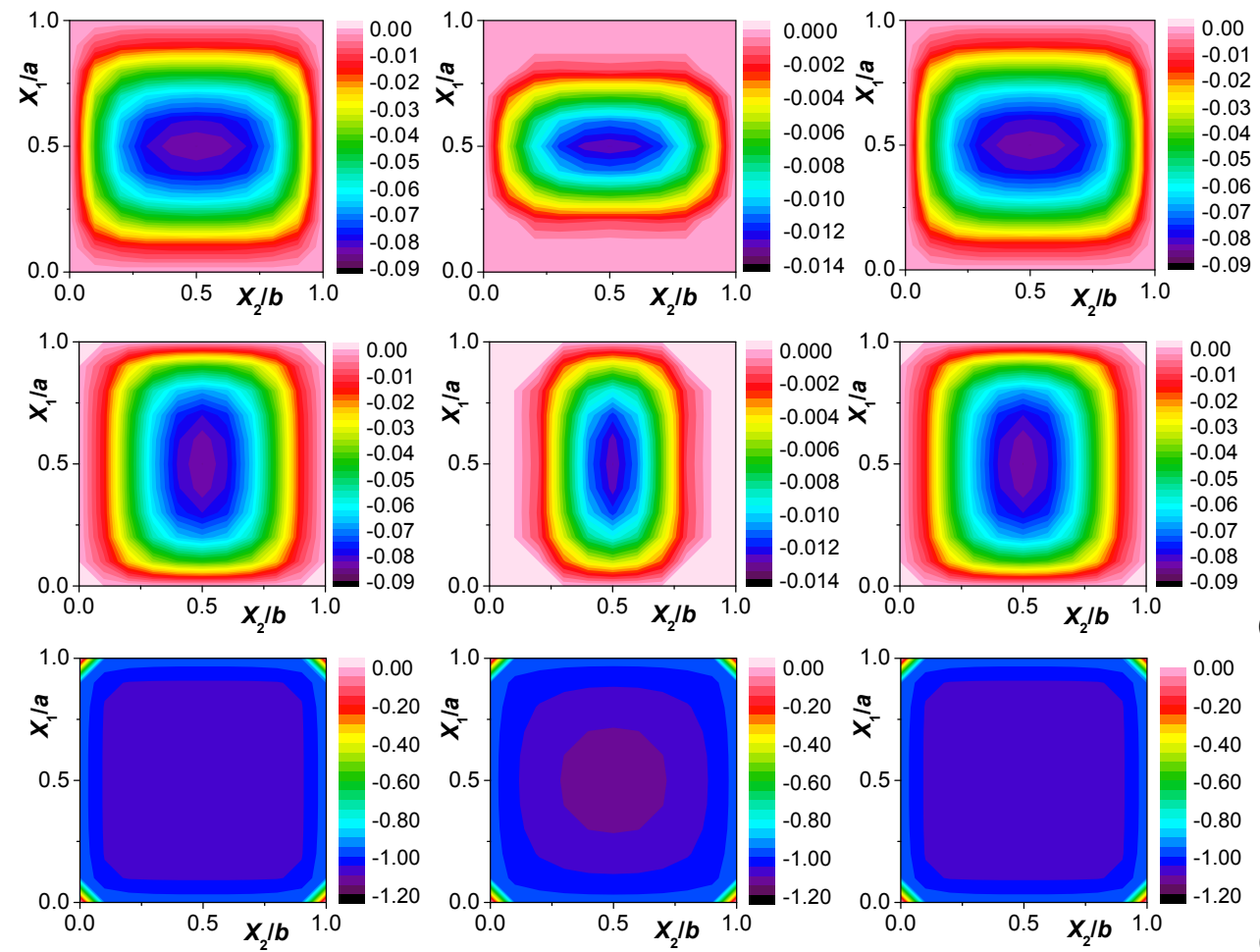

(b)
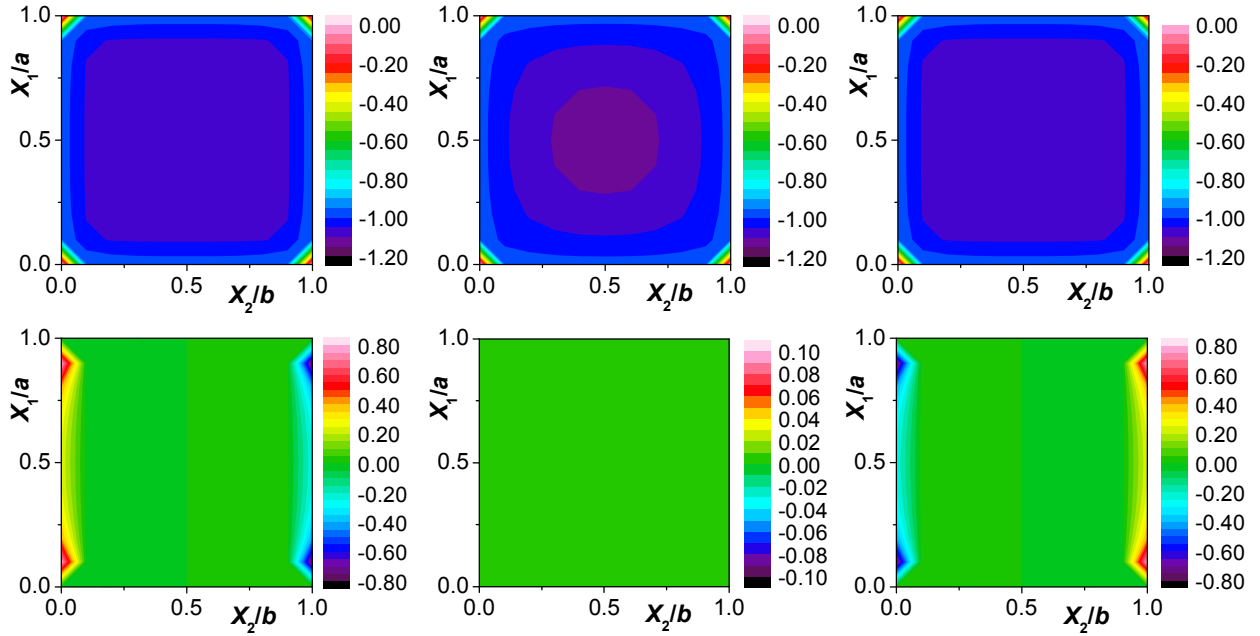

(c)
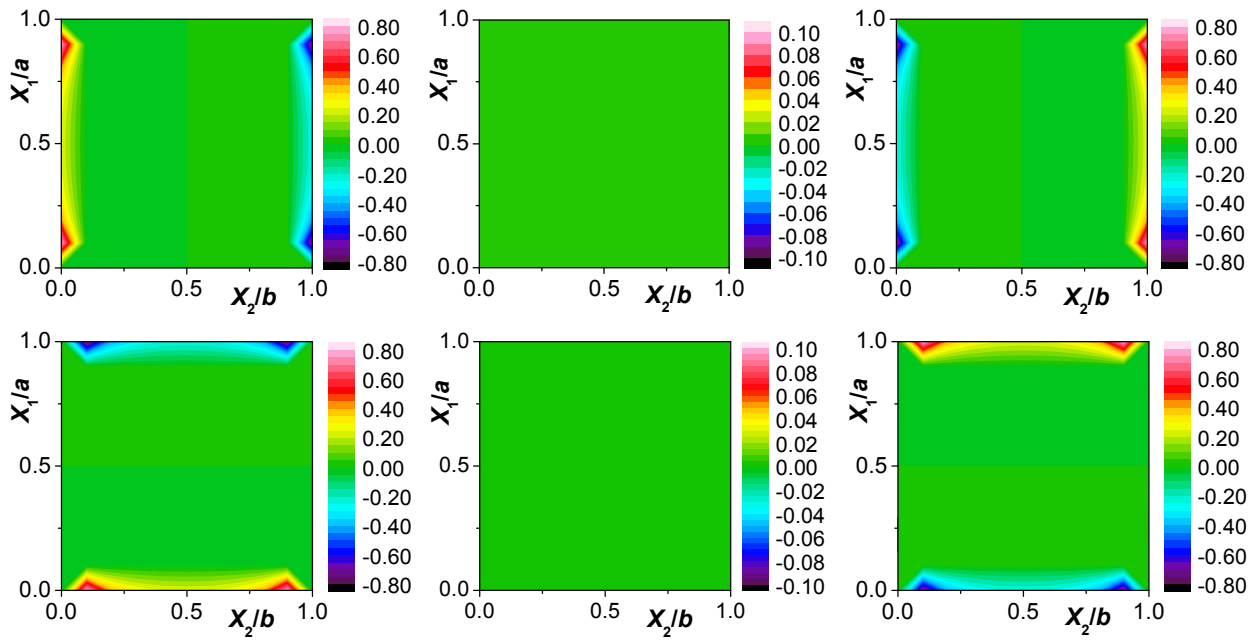

(d)
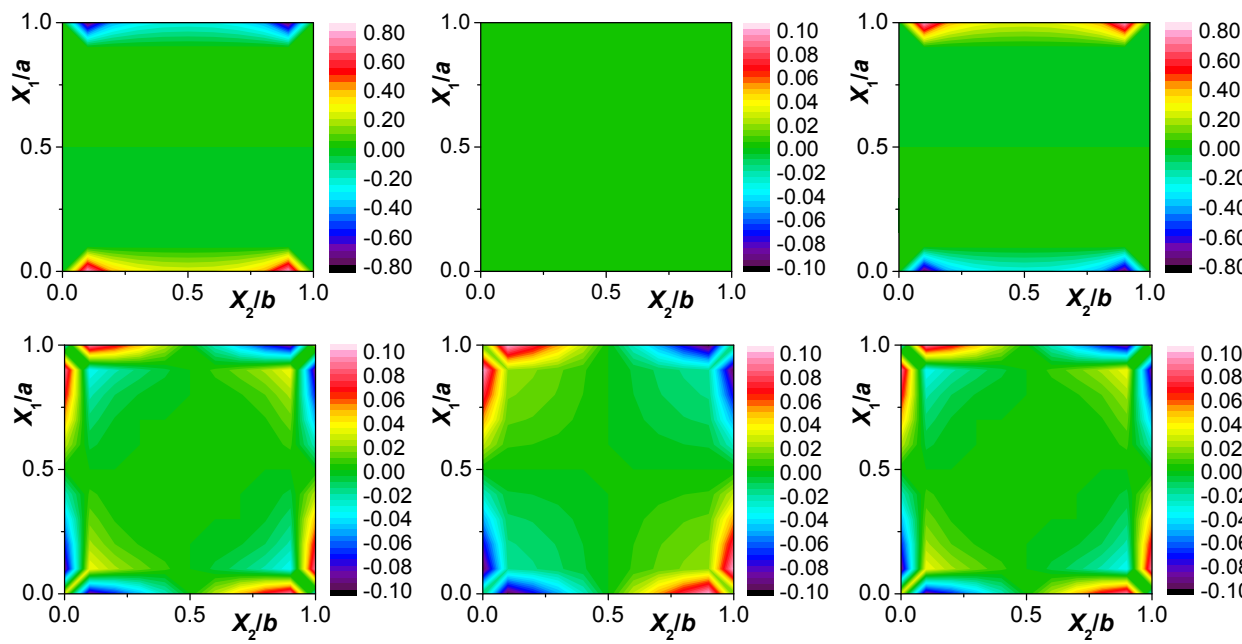

(e)

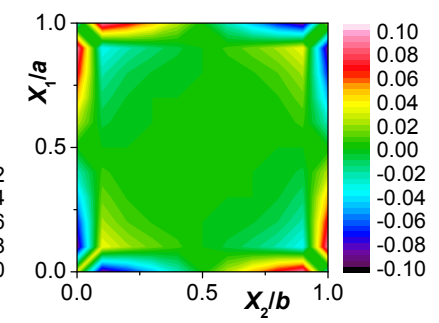

Fig. 2. $X_{1} X_{2}$ distributions of stress tensor components for the parallelepiped-shaped isotropic glass sample with the square cross-section and the width-to-height ratio equal to $1: 1$ : (a) $\sigma_{1}$, (b) $\sigma_{2}$, (c) $\sigma_{3}$, (d) $\sigma_{4}$, (e) $\sigma_{5}$, and (f) $\sigma_{6}$. The stress component values represent relative units with respect to $\sigma_{3}=-1$. Left, central and right columns correspond respectively to $X_{3}=0.2 c, 0.5 c$ and $0.8 c$. 

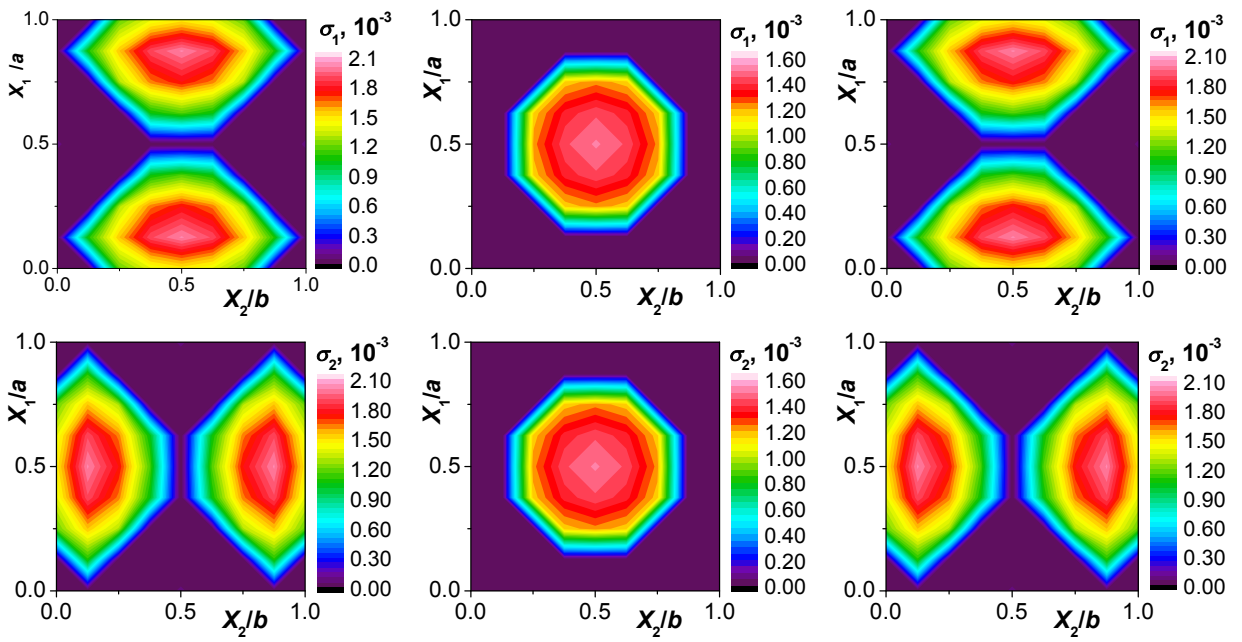

(a)
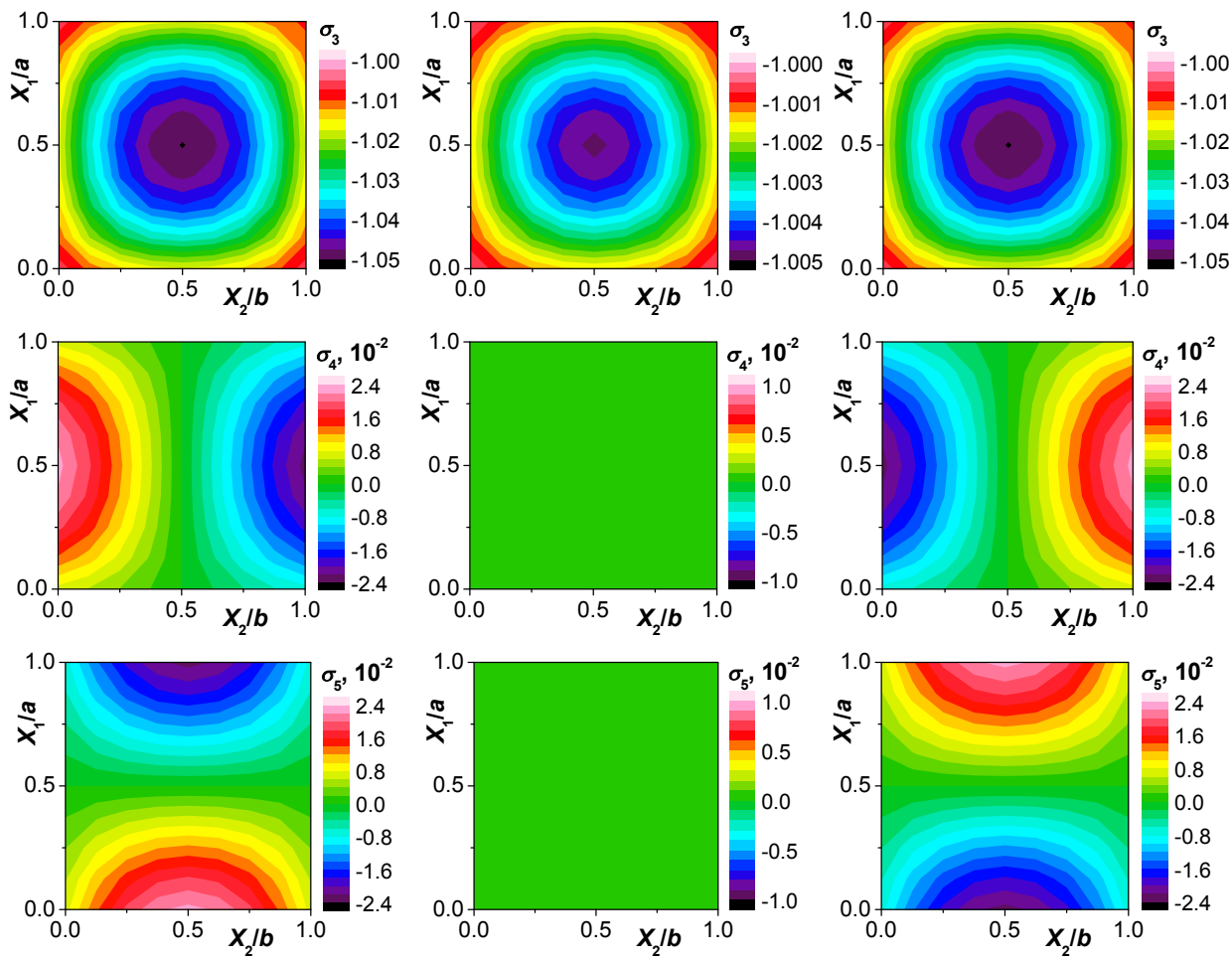

(d)
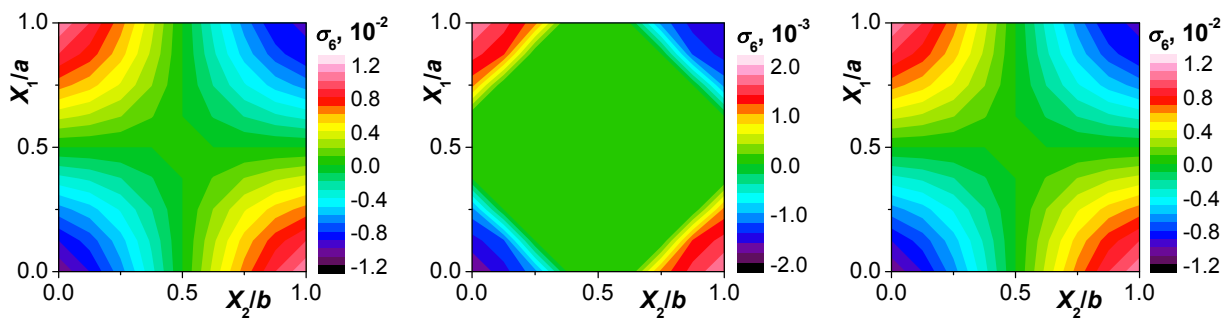

Fig. 3. $X_{1} X_{2}$ distributions of stress tensor components for the parallelepiped-shaped isotropic glass sample with the square cross-section and the width-to-height ratio equal to $1: 3$ : (a) $\sigma_{1}$, (b) $\sigma_{2}$, (c) $\sigma_{3}$, (d) $\sigma_{4}$, (e) $\sigma_{5}$, and (f) $\sigma_{6}$. The stress component values represent relative units with respect to $\sigma_{3}=-1$. Left, central and right columns correspond respectively to $X_{3}=0.2 c, c / 2$ and $0.8 c$. 

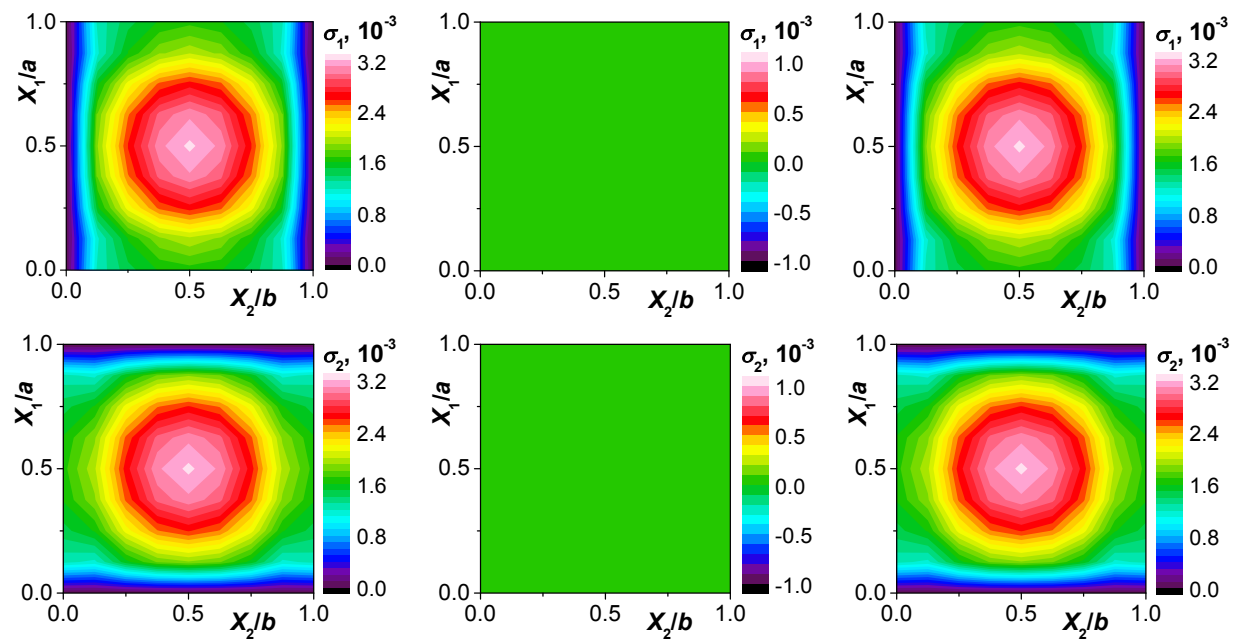

(a)
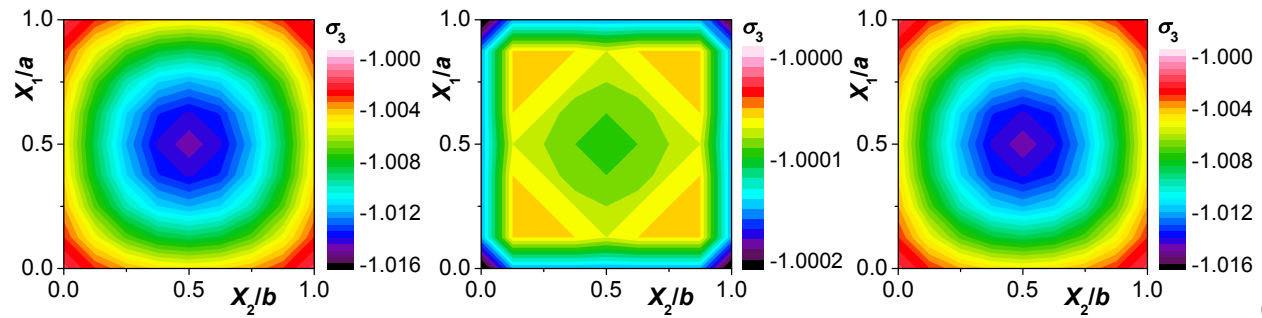

(b)
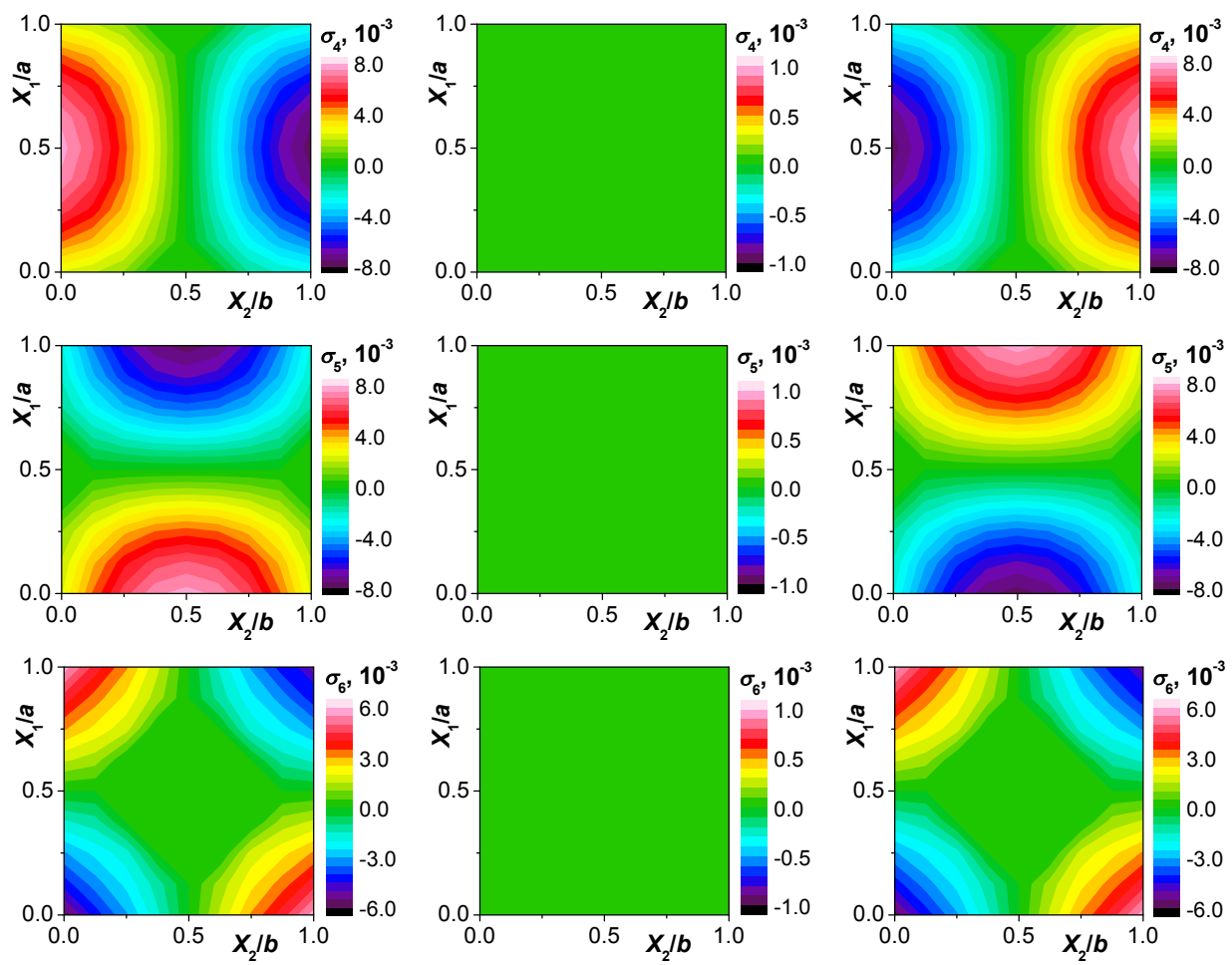

Fig. 4. $X_{1} X_{2}$ distributions of stress tensor components for the parallelepiped-shaped isotropic glass sample with the square cross-section and the width-to-height ratio equal to $1: 5$ : (a) $\sigma_{1}$, (b) $\sigma_{2}$, (c) $\sigma_{3}$, (d) $\sigma_{4}$, (e) $\sigma_{5}$, and (f) $\sigma_{6}$. The stress component value represent relative units with respect to $\sigma_{3}=-1$. Left, central and right columns correspond respectively to $X_{3}=0.2 c, 0.5 c$ and $0.8 c$. 
The shear stress components $\sigma_{4}$ and $\sigma_{5}$ become as large as $|0.8|$ in some parts of thin layers close to the lateral faces (at $X_{3}=0.2 c$ and $0.8 c-$ see Fig. $2 \mathrm{~d}$, e), though they are equal to zero in the central plane of the sample (at $X_{3}=0.5 c$ ). On the other hand, the component $\sigma_{6}$ reaches the value of $|0.1|$ even in the central cross-section, though it remains zero in the centre for all of the cross-sections (see Fig. 2f). The most important, the appearance of different components of the stress tensor would lead to incorrect determination of magnitude of the stress applied and, consequently, to the error occurring in determination of the piezooptic coefficients. Roughly speaking, the estimation error characteristic for the component $\sigma_{3}$ is equal to $\sim 12 \%$ for the sample with the width-to-height ratio equal to $1: 1$.

For the sample with the width-to-height ratio equal to $1: 3$, the stress tensor components $\sigma_{1}$ and $\sigma_{2}$ (see Fig. 3a, b) are equal to 0.0015 in the central cross-section (at $X_{3}=c / 2$ ) and reach the value of 0.002 at $X_{3}=0.2 c$. At the same time, the $\sigma_{3}$ component is equal to -1.005 at $X_{3}=c / 2$ and reaches the value of -1.05 at the upper and lower sample faces (at $X_{3}=0.2 c$ and 0 - see Fig. 3c). The shear components $\sigma_{4}$ and $\sigma_{5}$ are equal to $|0.025|$ near the lateral sample faces (at $X_{3}=0$ and $c$ ) and remain zero at $X_{3}=c / 2$ (see Fig. 3d, e). The component $\sigma_{6}$ is an order of magnitude smaller than the other shear components (see Fig. 3f). Since the maximum deviation of the actual stress from that applied to the sample is peculiar for the $\sigma_{3}$ component $(-1.05)$, the maximum error for the stress may be evaluated as $\sim 5 \%$. Notice that this error decreases by an order of magnitude in the central part of the sample, being equal only to $0.5 \%$. The latter value is surely not higher than the errors caused by various imperfections of the polarimetric or interferometric techniques.

Let us proceed to the sample with the width-to-height ratio equal to 1:5. In this case the stress tensor components $\sigma_{1}$ and $\sigma_{2}$ are zero in the central cross section (at $X_{3}=0.5 c$ ), though they become equal to 0.003 at $X_{3}=0.2 c$ and $0.8 c$ (see Fig. $4 \mathrm{a}, \mathrm{b}$ ). The $\sigma_{3}$ component is equal to 1.0001 at $X_{3}=0.5 c$ and -1.015 at $X_{3}=0.2 c$ and $0.8 c$ (see Fig. 4c). The shear stress components $\sigma_{4}$ and $\sigma_{5}$ are equal to $|0.008|$ near the lateral sample faces (at $X_{3}=0.2 c$ and $0.8 c$ ) and remain zero at $X_{3}=0.5 c$ (see Fig. $4 \mathrm{~d}$, e). Finally, the shear component $\sigma_{6}$ reaches its maximum equal to $|0.006|$ close to the upper and lower sample surfaces. Hence, the latter width-to-height ratio manifests the maximum stress error equal to $1.5 \%$. It is characteristic for the $\sigma_{3}$ component near the upper and lower sample faces. Nevertheless, since this error is some order of magnitude smaller in the central part of the sample, one can neglect it while determining the piezooptic coefficients.

As the last stage of our analysis, we have simulated the associated changes occurring in the optical phase difference due to inhomogeneous stress distribution. The phase difference is determined as $\delta(\Delta \Gamma)^{i j}=\Delta \Gamma_{\text {hom }}^{i j}-\Delta \Gamma_{\text {inh }}^{i j}$, where $\Delta \Gamma_{\text {hom }}^{i j}$ and $\Delta \Gamma_{\text {inh }}^{i j}$ are the total phase differences determined from relation $\Delta \Gamma_{\text {inh }}^{i j}=\arg \left(E_{1}^{\text {out }} / E_{3}^{\text {out }}\right)$, which follows from Eq. (33) respectively for the cases of homogeneous (the only constant nonzero component $\sigma_{3}=2.4 \times 10^{6} \mathrm{~N} / \mathrm{m}^{2}$ ) and inhomogeneous (all of the stress tensor components are nonzero) stress distributions. Fig. 5 
reproduces the spatial maps of the phase difference changes obtained for the samples having the width-to-height ratios equal to $1: 1,1: 3$ and 1:5. Even if the geometrical ratio is equal to $1: 1$, the optical indicatrix rotation angle within the homogeneous cells located near the upper and lower surfaces does not exceed $2 \mathrm{deg}$ and remains negligibly small in the central part of the sample.

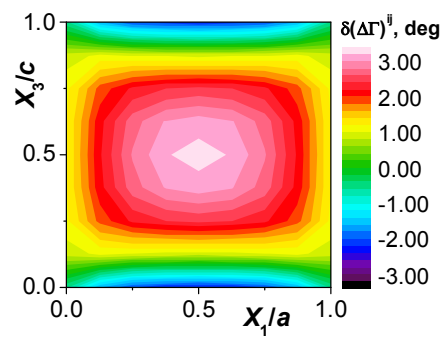

(a)

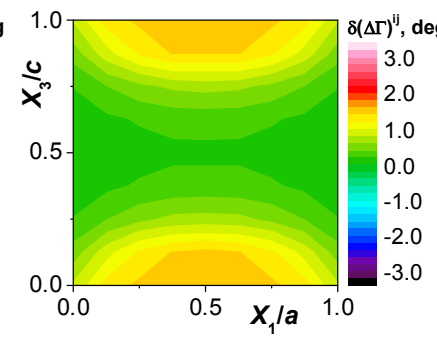

(b)

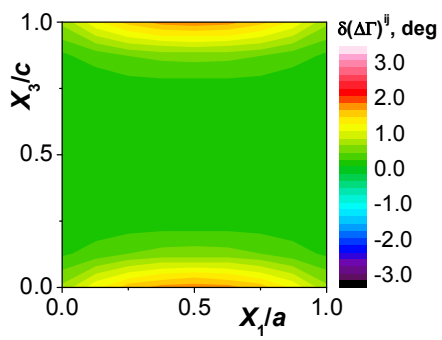

(c)

Fig. 5. $X_{1} X_{3}$ maps of phase difference changes, as simulated for the samples with the width-to-height ratios equal to $1: 1(a), 1: 3$ (b) and 1:5 (c).

In this respect let us remind that, for all of the three cases presented in Fig. 5, we have $\Delta \Gamma_{\text {hom }}^{i j}=39 \mathrm{deg}$ at the sample thickness equal to $10^{-2} \mathrm{~m}$. When the beam propagates through the centre of the $X_{1} X_{3}$ cross-section of the sample, the relative errors for the phase difference are equal to $8.4,0.4$ and $0.00025 \%$ for the geometrical ratios 1:1, 1:3 and 1:5, respectively. Even in the worst case, when one considers whole cross-section of the sample under the condition that the ratio is equal or less than 1:3, the maximal error for the phase difference does not exceed $3.8 \%$.

\section{Conclusions}

In this work we have demonstrated that the error of experimental determination of the piezooptic coefficients, which is caused by friction appearing between the sample surfaces and the substrates, can be eliminated by choosing properly the geometrical parameters of the sample. In the case of initially isotropic samples with the square cross-section, the width-to-height ratio has to be equal or smaller than 1:3. This reduces the error for the phase difference down to $3.8 \%$ provided that the absolute value of the phase difference is equal to $39 \mathrm{deg}$. It is worth noticing that our conclusion agrees well with the experimentally based data presented in Ref. [4]. Moreover, if one employs a probing optical beam with the diameter $\sim 2 \mathrm{~mm}$, this error could be still further reduced (at least to $0.7 \%$ ) under the condition that the sample height equals to $9 \mathrm{~mm}$. Hence, the main remaining problems of the piezooptic experiments are accurate application of mechanical stresses, precise alignment of samples and preparation of samples with parallel enough faces.

\section{References}

1. Narasimhamurty T S. Photoelastic and electrooptic properties of crystals. New York: Plenum Press (1981).

2. Sirotin Yu I and Shaskolskaya M P. Fundamentals of crystal physics. Moscow: Nauka (1979).

3. Vlokh O G. Spatial dispersion phenomena in parametric crystal optics. Lviv: Vyshcha Shkola (1984).

4. Frocht M M, Photoelasticity. N. Y.: John Wiley (1946).

5. Kemp James C, 1969. Piezo-optical birefringence modulators: new use for a long-known effect. J. Opt. Soc. Amer. 59: 950-954. 
6. Grakh I I and Mozhanskaya A F, 1971. A type of mechanically anisotropic, optically sensitive material. Mekhanika Polimerov. 5: 835-839.

7. Weber Y-J, 1995. Determination of internal strain by optical measurements. Phys. Rev. B. 51: 12209-12215.

8. Hammer H. and Lionheart W R B, 2005. Reconstruction of spatially inhomogeneous dielectric tensors through optical tomography. J. Opt. Soc. Amer. A. 22: 250-255.

9. Puro A É and Karov D D, 2007. Tensor field tomography of residual stresses. Opt. Spectrosc. 103: 678-682.

10. Aben H, Errapart A, Ainola L and Anton J, 2005. Photoelastic tomography for residual stress measurement in glass. Opt. Eng. 44: 093601.

11. Balakshii V I, Parygin V N and Chirkov L E. Physical fundamentals of acoustooptics. Moscow: Radio i Sviaz' (1985).

12. $\mathrm{Xu} \mathrm{J}$ and Stroud R. Acousto-optic devices: principles, design, and applications. New York: Wiley (1992).

13. Mys O, Kostyrko V, Smyk M, Krupych O and Vlokh R, 2014. Anisotropy of acoustooptic figure of merit in optically isotropic media. Appl. Opt. 53: 4616-4627.

14. Dixon R W, Cohen M G, 1966. A new technique for measuring magnitudes of photoelastic tensors and its application to lithium niobate. Appl. Phys. Lett. 8: 205-207.

15. Dixon R W, 1967. Photoelastic properties of selected materials and their relevance for application to acoustic light modulators and scanners. J. Appl. Phys. 38: 5149-5153.

16. Papadakis E, 1967. Ultrasonic phase velocity by the pulse-echo-overlap method incorporating diffraction phase corrections. J. Acoust. Soc. Amer. 42: 1045-1051.

17. Vasylkiv Yu, Kvasnyuk O, Krupych O, Mys O, Maksymuk O and Vlokh R, 2009. Reconstruction of 3D stress fields basing on piezo-optic experiment. Ukr. J. Phys. Opt. 10: 22-37.

18. Krupych O, Savaryn V and Vlokh R, 2014. Precise determination of full matrix of piezo-optic coefficients with a four-point bending technique: the example of lithium niobate crystals. Appl. Opt. 53: B1-B7.

19. Krupych O, Savaryn V, Krupych A, Klymiv I and Vlokh R, 2013. Determination of piezooptic coefficients of crystals by means of four-point bending. Appl. Opt. 52: 4054-4061.

20. Skab I, Smaga I, Savaryn V, Vasylkiv Yu and Vlokh R, 2011. Torsion method for measuring piezooptic coefficients. Cryst. Res. Technol. 46: 23-36.

21. Vasylkiv Y, Savaryn V, Smaga I, Skab I and Vlokh R, 2011. On determination of sign of the piezooptic coefficients using torsion method. Appl. Opt. 50: 2512-2518.

22. http://hypertextbook.com/facts/2005/glass.shtml

23. Bayda E N. Some spatial problems of the theory of elasticity. Leningrad: Izd. LGU (1983).

Kvasnyuk O., Vasylkiv Yu., Krupych O. and Vlokh R. 2014. Preferable geometrical parameters of samples for piezooptic experiments. Ukr.J.Phys.Opt. 15: 195 - 206.

Анотація. У роботі показано, що похибку експериментального визначення n'єзооптичних коефіцієнтів, спричинену силами тертя між поверхнями зразка $i$ підкладками, можна усунути належним підбором геометричних параметрів зразка. Відношення ширини зразка до його висоти повинно дорівнювати або бути мениим за 1:3. У такому разі похибку, спричинену бочкоподібною дисторсією, можна зменшити приблизно до 1\%. 